\title{
Thermal Modeling of Cooldown Processes In Portable HPGe Spectrometers
}

\author{
O. Yakovlev, V. Malgin and Y. Viba
}

\begin{abstract}
The Stirling cycle cryocoolers are usually used for HPGe detectors cooling in $\gamma$-radiation portable spectrometers for field applications. The cooling of large size germanium detectors up to cryogenic temperatures by application of low-power cryocoolers makes the topical task of the thermal losses analysis. The equivalent thermal model of detector unit and Stirling cryocooler created by thermoelectric analogies is presented. Thermal modeling is used for analysis and optimization of cooling path from cryocooler to detector can for thermal losses reduction. The calculated cooldown curves of the detector unit as the transition process for the proposed model are compared to the experimental results. It is shown that the proposed model allows simulate the cooldown processes for HPGe detector with accuracy sufficient for the practical applications. The temperature distribution diagrams for the thermal joints obtained by SolidWorks Simulation are presented.
\end{abstract}

Keywords- Cooldown curve, HPGe detector, Stirling cryocooler, Thermoelectric analogy.

\section{INTRODUCTION}

Portable gamma-ray spectrometers with High Purity Germanium (HPGe) detectors cooled by Stirling cryocoolers are widely used for the in-situ radioactive monitoring applications [1-4]. To ensure the maximum operating time under field conditions without battery recharging low-power Stirling cryocooler should be used. The low cooling power (typically 1-3 W) limits the permissible heat losses in the chamber defined by the construction of cryostat and level of vacuum. When the detector is cooled, sufficient vacuum is provided by a zeolite (molecular sieve) adsorbing residual gases at operating temperatures which are in the range of $80 \mathrm{~K}$. The operation of portable devices is characterized by long periods of storage or transportation in the OFF state when the detector unit is in warming up state. During that state the zeolite releases the adsorbed gas molecules. Vacuum decrease increases the heat losses in the chamber for cooldown process. In a critical case the cooling power of cryocooler is not enough to provide HPGe detector cooling to the required temperature $[2,3]$.

Therefore, simulation and analysis of the heat losses in the

Manuscript received January 12. 2017.

Oleg Yakovlev is PhD Student at Riga Technical University, Riga, Latvia and mechanical design engineer at Baltic Scientific Instruments SIA, Riga, Latvia. (e-mail: o.jakovlev@bsi.lv).

Vladislav Malgin is researcher at Baltic Scientific Instruments SIA, Riga, Latvia.

Yanis Viba is Professor at Riga Technical University, Riga, Latvia. vacuum chamber become to be the most important.

The total heat transfer from the chamber walls is a sum of the conduction through supports and current leads, residual gas conduction and radiation [5].

- Heat transfer through supports and current leads. For the temperatures in range $80 \mathrm{~K}-300 \mathrm{~K}$ the thermal conductivity of solids may be considered as constant and heat transfer proportional to the difference between temperatures of outer shell and cooled units.

- Heat transfer by residual gas. As usual the residual gas pressure in cryostat is in range of $10^{-4}-10^{-3}$ mbar and molecules free path is more than a distance between surfaces. In according to the Knudsen equation for region of "free-molecule" conduction the heat transfer is proportional to the pressure and the temperature difference.

- Heat transfer by thermal radiation. In according to the Stefan-Boltzmann equation the heat emission from surface of body is proportional to it temperature of degree 4 .

It should be underlined that in distinct from conduction through supports and current leads the gas conduction and radiation transfer may be estimated with rather less accuracy. The reason is the difficulty of determining the accommodation coefficient and emissivity, respectively. However, it is usually possible to estimate an upper bond for the heat transfer by these processes and it is sufficient to meet the required demands.

To reduce the heat transfer by thermal radiation a radiation shields are usually used. So, in case of high vacuum, the thermal conductivity of supports contributes mainly to the total sum of heat transfer.

In case of decreased vacuum in cryostat after warming up the molecular conduction of residual gas, the dominating heat load contribution during the cooldown process is introduced. The heat transfer due to thermal radiation should be taken into account but it is not determinative in that situation [6].

The existing dependency of total heat losses in the cryostat during its cooling after heating from temperature difference allows to use the thermo-electrical analogies method for thermal calculation. This method allows to evaluate the thermal characteristics of the spectrometer's cryostat through the analysis of equivalent electrical circuit. The method provides accuracy sufficient for engineering applications, while retaining a clear physical meaning of the processes. 


\section{Thermal MOdeling OF CRYOCOOLER's OUtPut CHARACTERISTICS}

Stirling cryocooler performance is characterized by cooling power and cooldown characteristics specified in the data sheet, Fig.1. Based on these characteristics the cryocooler with sufficient accuracy for practical thermal calculations can be represented as a two-terminal circuit consisting of a current source $I_{0}$, and elements $R_{0}, C_{0}$ which simulate the heat losses and heat capacity of cold finger, Fig.2. These parameters can be easily calculated from the standard characteristics in the data sheet.

TABLE I: THE THERMO-ELECTRIC ANALOGOUS QUANTITIES

\begin{tabular}{|c|c|c|c|}
\hline \multicolumn{2}{|c|}{ Thermal quantities } & \multicolumn{2}{c|}{ Electric quantities } \\
\hline Heat transfer rate & $\mathrm{P},(\mathrm{W})$ & Current & $\mathrm{I},(\mathrm{A})$ \\
\hline Temperature & $\Theta,(\mathrm{K})$ & Voltage & $\mathrm{U},(\mathrm{V})$ \\
\hline Th. resistance & $\mathrm{R},(\mathrm{K} / \mathrm{W})$ & Resistance & $\mathrm{R},(\mathrm{Ohm})$ \\
\hline Thermal capacitance & $\mathrm{C},(\mathrm{J} / \mathrm{K})$ & Capacity & $\mathrm{C},(\mathrm{F})$ \\
\hline
\end{tabular}

The output voltage $U_{\text {out }}$ is the equivalent of a cold tip temperature and corresponds to cooling power $I_{c}$, at ambient reject temperature $U_{0}$, as follows:

$$
U_{\text {out }}=U_{0}-R_{0} \cdot\left(I_{0}-I_{c}\right)
$$

For example, the cryocooler FPFD, with characteristics given in [9], for input power $40 \mathrm{~W}$ and ambient reject
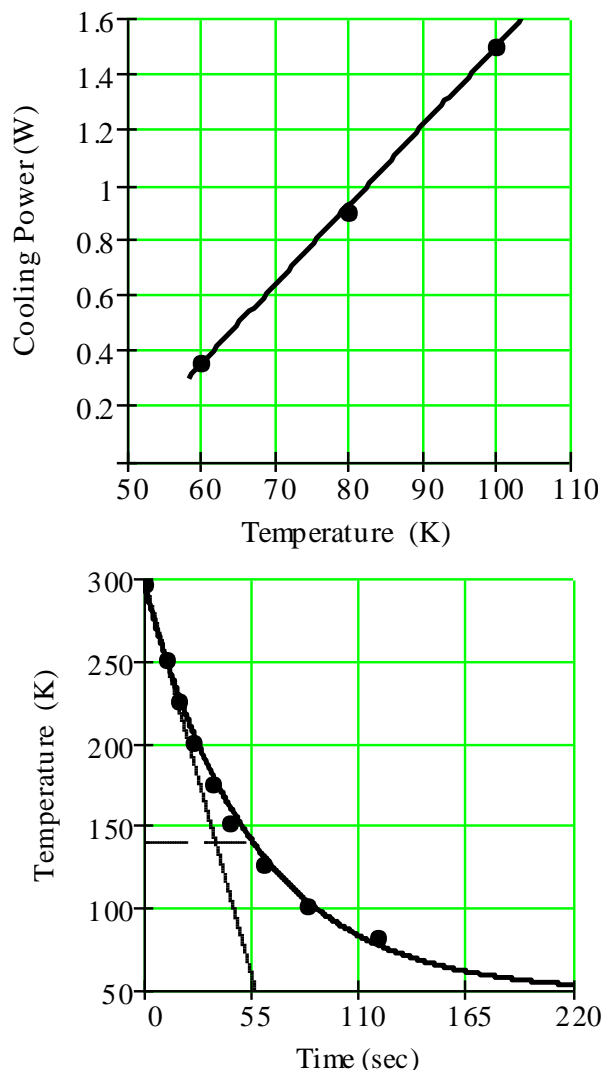

Fig.1 Example: cooling power (upper) and cooldown characteristic (lower) of cryocooler FPFD for ambient reject temperature $23^{0} \mathrm{C}$ presented in [9].

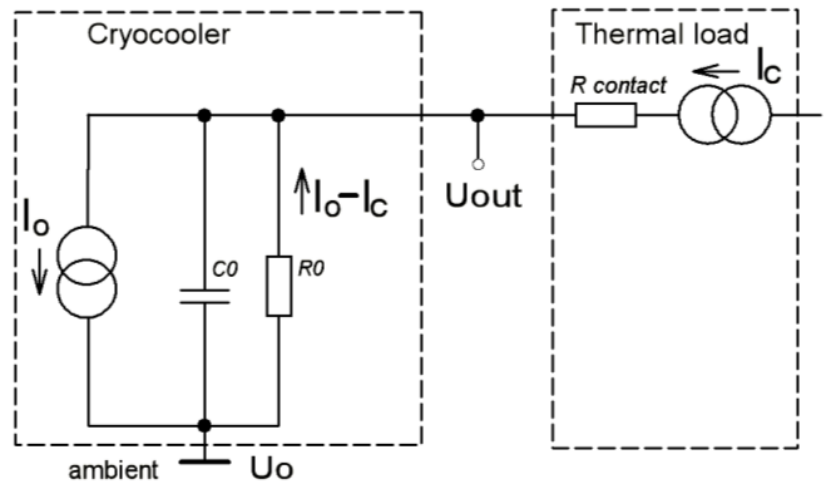

Fig.2 Equivalent circuit of cryocooler used for thermal modeling.

temperature $U_{0}=296 \mathrm{~K}$, provides a cooling power $I_{c l}=0.35 \mathrm{~W}$ at a temperature at the cold tip $U_{\text {out }}=60 \mathrm{~K}$ and $I_{c 2}=0.9 \mathrm{~W}$ when temperature increases to $U_{\text {out } 2}=80 \mathrm{~K}$. So, the system of equations can be formulated from (1) and it solution gives values of the parameters $R_{0}$ and $I_{0}$.

$$
\begin{aligned}
& R_{0}=\left(U_{\text {out } 2}-U_{\text {out1 }}\right) /\left(I_{c 2}-I_{c 1}\right)=36,36 \mathrm{~K} / \mathrm{W} \\
& I_{0}=I_{C 1}+\left(U_{0}-U_{\text {out } 1}\right) / R_{0}=6,84 \mathrm{~W}
\end{aligned}
$$

Note that the physical meaning of the parameter $I_{0}$, within this simplified model, corresponds to a Carnot cycle thermal power for a given cryocooler.

Parameter $C_{0}$ corresponding to equivalent thermal capacity of cold finger is calculated through the time constant from cooldown curve without a load. For example, the cooldown curve from $296 \mathrm{~K}$ to $50 \mathrm{~K}$ for cryocooler FPFD without load is shown in [9]. Therefore, the time constant, i.e. time during which the amplitude of process decreases in e-times (from $296 \mathrm{~K}$ to $140,5 \mathrm{~K})$ is $\tau=56 \mathrm{sec}$. So,

$$
C_{0}=\tau / R_{0}=1,54 \mathrm{~J} / \mathrm{K}
$$

On Fig. 1,b the points correspond to experimental cooldown characteristic for FPFD cryocooler [9]. The line corresponds to calculated exponential function (4) with parameters mentioned above.

$$
U_{\text {out }}(t)=U_{0}-I_{0} \cdot R_{0} \cdot\left[1-\exp \left(-t / R_{0} \cdot C_{0}\right)\right]
$$

It should be mentioned that the calculated values of parameters are valid only for specified values of input power and ambient reject temperature. When input power or ambient temperature are changed the load characteristics of cryocooler and parameters of thermal model are changed too.

On Fig. 3 the load characteristics for various models of Stirling cryocoolers are presented. The points correspond to experimental data presented in [7-10], and the lines are calculated by application of proposed model. These examples demonstrate that proposed equivalent model of cryocooler has accuracy sufficient for engineering calculations in a wide range of output cooling power and cooling temperatures. 


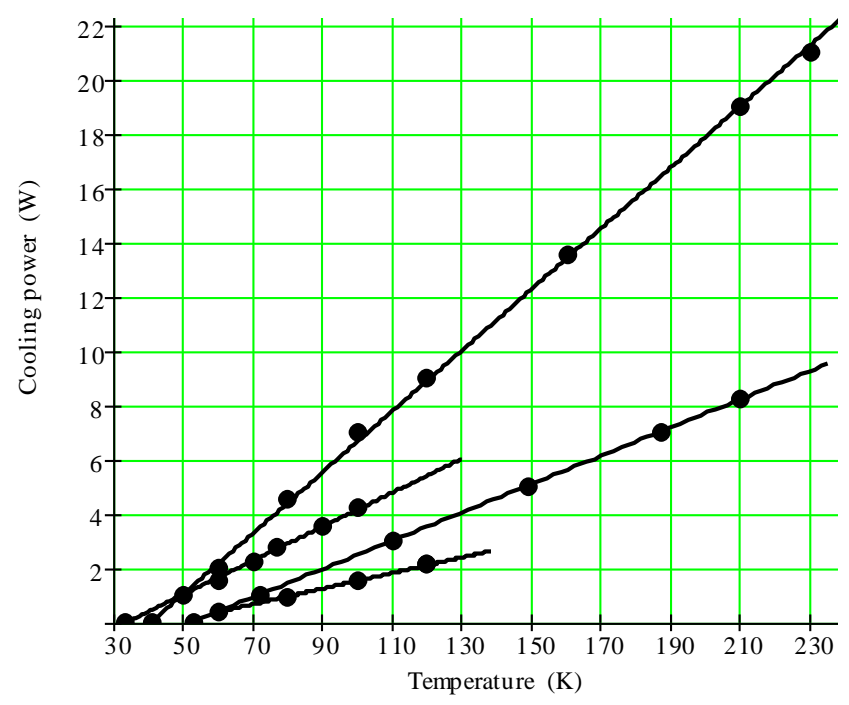

Fig.3 Cooling power for cryocoolers: 1- LPT9310, Thales [8]; 2 - CryoTel DT60, Sunpower [9]; 3- SF400, AIM [10]; 4-FPFD, Korea [7].

\section{Math Thermal Model of HPGe Detector Unit}

Typical design of the cryostat with a HPGe detector and cryocooler showing the basic units having the most significant impact on cooling performance is presented on Fig.4. Undoubtedly the equivalent circuit presented in Fig. 5 in according with TEAM is simplified, but allows to evaluate the basic processes that affect to the rate of cooling. The thermal parameters of the cryostat's units and related components of the equivalent electrical circuit are shown in Table II.

\begin{tabular}{|c|c|}
\hline TABLEol & Thermal elements of detector unit \\
\hline$R 0, C 0$ & Equivalent output thermal parameters of cryocooler \\
\hline$R 1, R 3$ & $\begin{array}{c}\text { Equivalent thermal resistance by gas conduction and } \\
\text { radiation between containers shell and vacuum can }\end{array}$ \\
\hline$C 1$ & Thermal capacity of copper container with zeolite \\
\hline$R 2$ & Thermal resistance of thermal strap \\
\hline$C 2$ & Thermal capacity of container with HPGe detector \\
\hline$R 4$ & Thermal resistance between container and Ge crystal \\
\hline$C 3$ & Thermal capacity of the germanium crystal \\
\hline
\end{tabular}

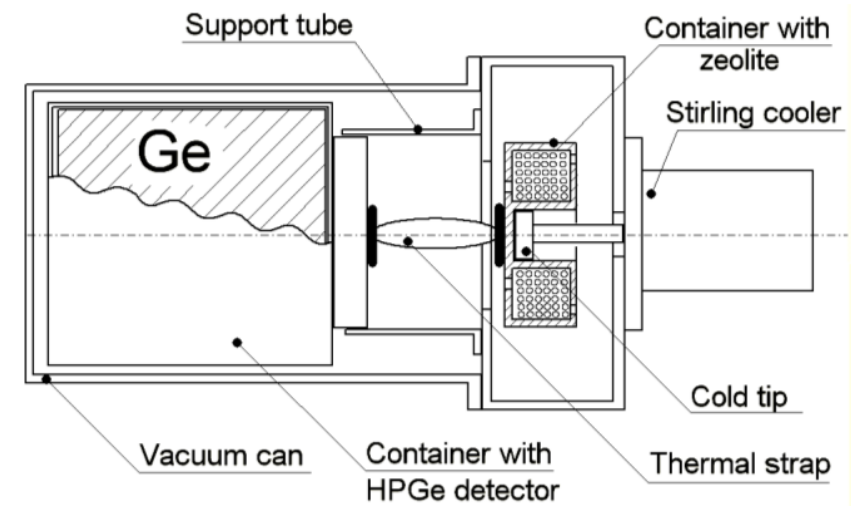

Fig.4. Typical design of HPGe detector unit with cryocooler

It follows from thermo-electrical analogies that thermal resistance of element equals thermal transfer through it divided to a temperature drop. In general, the elements $R 1$ and $R 3$ related to the total heat losses $Q_{\text {total }}$ in the vacuum chamber have a nonlinear dependence from both residual gas pressure $p$, and temperature difference between the outer shell of cryostat $T_{1}$, and cooled parts $T_{2}$.

$$
Q_{\text {total }}=Q_{\text {cond }}+Q_{\text {resid }}+Q_{\text {rad }}
$$

where:

$Q_{\text {cond }} \propto\left(T_{1}-T_{2}\right)$ - heat transfer through supports and leads; $Q_{\text {resid }} \propto p \cdot\left(T_{1}-T_{2}\right)$ - heat transfer by residual gas in region of molecular conductance;

$Q_{\text {rad }} \propto\left(T_{1}^{4}-T_{2}^{4}\right)$ - heat transfer by thermal radiation.

This nonlinear dependence is determined by contribution of heat transfer by components $Q_{\text {resid }}$ and $Q_{\text {rad }}$.

Note, the degree of non-linearity depends on the actual relationships between components of heat losses mentioned above, which are individual for each type of cryostats.

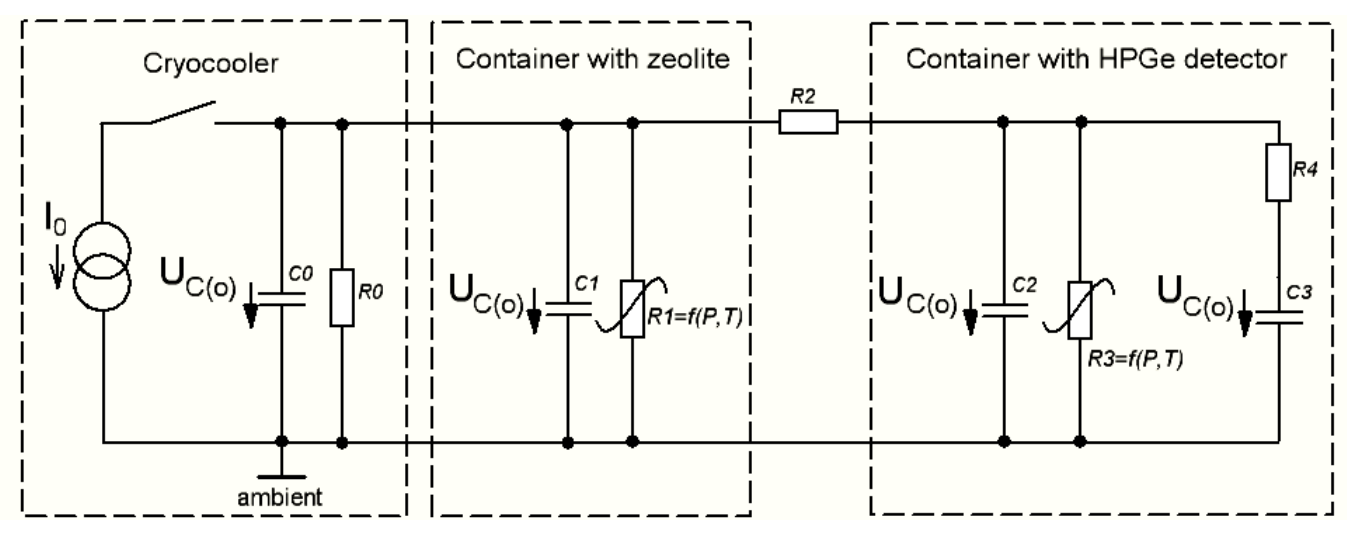

Fig.5 Equivalent circuit of cryostat with cooler used for thermal modeling. 


\section{CoOling as a Transient Process}

In according with equivalent circuit shown in Fig. 5 the cooling process of germanium crystal corresponds to charging of capacitor $C_{3}$ by current source $I_{0}$. Neglecting the changes in pressure of residual gases and nonlinearity of heat losses (assume that values $R 1$ and $R 3$ are constant) cooling can be presented as the transition process in equivalent circuit.

For the layout a cryostat shown in Fig. 4 the elements of equivalent circuit related to output parameters of cryocooler and a container with zeolite are connected in parallel and can be united into one. So,

$$
C_{01}=C_{0}+C_{1} \text { and } R_{01}=R_{0} \cdot R_{1} /\left(R_{0}+R_{1}\right)
$$

Note, if container with zeolite is affixed to container with germanium detector the related elements of equivalent circuit are connected in parallel and can be united into one, also.

In this case, the equivalent circuit includes only three main elements for storage of thermal energy. There are: cold finger of cryocooler and container with zeolite, jointly; container for germanium detector and germanium crystal, each separately. This separation is important because the detector cooling occurs through the thermal contact with a container having high thermal resistance $R 4$.

The voltage on each capacitor $U_{C i}(t)$, corresponding to the temperature of the related cryostat's part can be written as:

$$
U_{C i}(t)=U_{0}-\left[U_{C, i}(\infty)+U_{T r, i}(t)\right],
$$

where:

$U_{0}$ - is a base potential related to ambient reject temperature;

$U_{C, i}(\infty)$ - is a steady state voltage on i-capacitor related to final temperature after cooling;

$U_{T r, i}(t)$ - is a transient stage voltage on capacitor;

$$
U_{T r, i}(t)=A_{i, 1} \cdot e^{\lambda_{1} \cdot t}+A_{i, 2} \cdot e^{\lambda_{2} \cdot t}+A_{i, 3} \cdot e^{\lambda_{3} \cdot t},
$$

where:

$A_{i, 1} ; A_{i, 2} ; A_{i, 3}$ - are the constants of transient stage for icapacitor;

$\lambda_{1} ; \lambda_{2} ; \lambda_{3}-$ the roots of characteristic equation;

$t$ - the current time.

The steady state voltages on capacitors related to final temperatures after cooling can be found by Kirchhoff's law and equals:

$$
\begin{array}{r}
U_{C 1}(\infty)=I_{0} \cdot \frac{R_{01} \cdot\left(R_{2}+R_{3}\right)}{R_{01}+R_{2}+R_{3}} \\
U_{C 2}(\infty)=U_{C 3}(\infty)=I_{0} \cdot \frac{R_{01} \cdot R_{3}}{R_{01}+R_{2}+R_{3}}
\end{array}
$$

Determination of characteristic equation for equivalent circuit, Fig.5 (the cooling process in the detector unit) is a standard procedure from the theory of electrical circuits (see for example [11]) and in this article is not described. The system of algebraic equations for determining the coefficients $A_{i, 1} ; A_{i, 2} ; A_{i, 3}$ based on the initial conditions of transient process can be expressed from equations (7) and (8) as follows:

$$
\left\{\begin{array}{l}
U_{C i}(0)=U_{C, i}(\infty)+A_{i, 1}+A_{i, 2}+A_{i, 3} \\
U_{C i}^{\prime}(0)=\lambda_{1} \cdot A_{i, 1}+\lambda_{2} \cdot A_{i, 2}+\lambda_{3} \cdot A_{i, 3} \\
U_{C i}^{\prime \prime}(0)=\lambda_{1}^{2} \cdot A_{i, 1}+\lambda_{2}^{2} \cdot A_{i, 2}+\lambda_{3}^{2} \cdot A_{i, 3}
\end{array}\right.
$$

TABLE III: THE INITIAL CONDITIONS OF TRANSIENT STAGES

\begin{tabular}{|c|c|c|c|}
\hline & $U_{C i}(0)$ & $U_{C i}^{\prime}(0)$ & $U_{C i}^{\prime \prime}(0)$ \\
\hline $\mathrm{i}=1$ & 0 & $-\frac{I_{0}}{C_{0}}$ & $I_{0} \cdot \frac{R_{0}+R_{1}}{R_{0} \cdot R_{1} \cdot C_{0}^{2}}$ \\
\hline $\mathrm{i}=2$ & 0 & 0 & $\frac{-I_{0}}{R_{1} \cdot C_{0} \cdot C_{1}}$ \\
\hline $\mathrm{i}=3$ & 0 & 0 & 0 \\
\hline
\end{tabular}

The values of the initial conditions for transition process are based on the following:

- at the beginning of transition process the voltages on the capacitors equals to zero. The physical meaning of this is that parts of the cryostat are in thermal equilibrium with the surrounding environment;

- at the initial moment of transition process the capacitor is equivalent to a short circuit;

- the second derivatives of voltage change on the capacitors are determined based on the rate of increase in charge current.

Note that the analytic solution of transition process is valid under assumption that all parameters of equivalent circuit are constant. In a large range of temperature changes during cooling from $300 \mathrm{~K}$ up to $90 \mathrm{~K}$ this is not performed. So, an average value is used. However, the analysis of analytical solutions allows us to consider the relationship between the main processes that occur during cooling of the cryostat.

Undoubtedly, the further use of modern software packages for thermal calculations will provide a more accurate temperature distribution during the cooling process. In this case the presence of an analytic solution can be used for the testing of more complex model. 


\section{NuMERICAL SimulATION AND EXPERIMENT}

The proposed model was used to calculate the detector's cooling process of the portable spectrometer HandSPEC produced by Baltic Scientific Instruments SIA from Latvia. The values of the model parameters were determined based on the design of the given detector unit.

The tested device has applied the Stirling cryocooler of the cooling power 1W @ 80K. Its equivalent parameters $R_{0}, C_{0}$ were calculated from (2) - (4) and based on a load characteristic measured experimentally. The heat load was set by a separate resistive heater. The values of $C_{1}, C_{2}$, and $C_{3}$ are equal to the heat capacities of the most massive components - copper container for zeolite, aluminum container for HPGe detector and HPGe detector, directly. The specific heats for copper, aluminum and germanium are temperature dependent. So, the average values for temperature range $90 \mathrm{~K}-300 \mathrm{~K}$ are used. The value of the thermal resistance $R_{2}$ for copper strap linked cold tip with the container for HPGe detector was calculated by Solidworks Simulation (see below). The values of $R_{l}$ and $R_{3}$ have been chosen based on the total heat losses in a cryostat which had reached $1.1 \mathrm{~W}$ for temperature $90 \mathrm{~K}$. The parameters of equivalent model for cryostat of HandSPEC spectrometer are shown in Table 4. In according to (7) - (11) the transient process was calculated. The cooldown curves for cryostat components are plotted and are shown in Fig. 6. Undoubtedly these curves are valid before entering to the closed loop controllable mode when the target temperature is reached.

Obtained cooling curves allow to evaluate the difference in cooling rate of the main components of cryostat. In reality, a temperature sensor (thermistor PT100 or silicon diode) are mounted on the container for the HPGe detector as usual. Therefore, it should be taken into account that there is a delay in the cooling of germanium crystal relative to temperature readings on the display. For HandSPEC spectrometer this delay goes up to one hour, Fig.6.

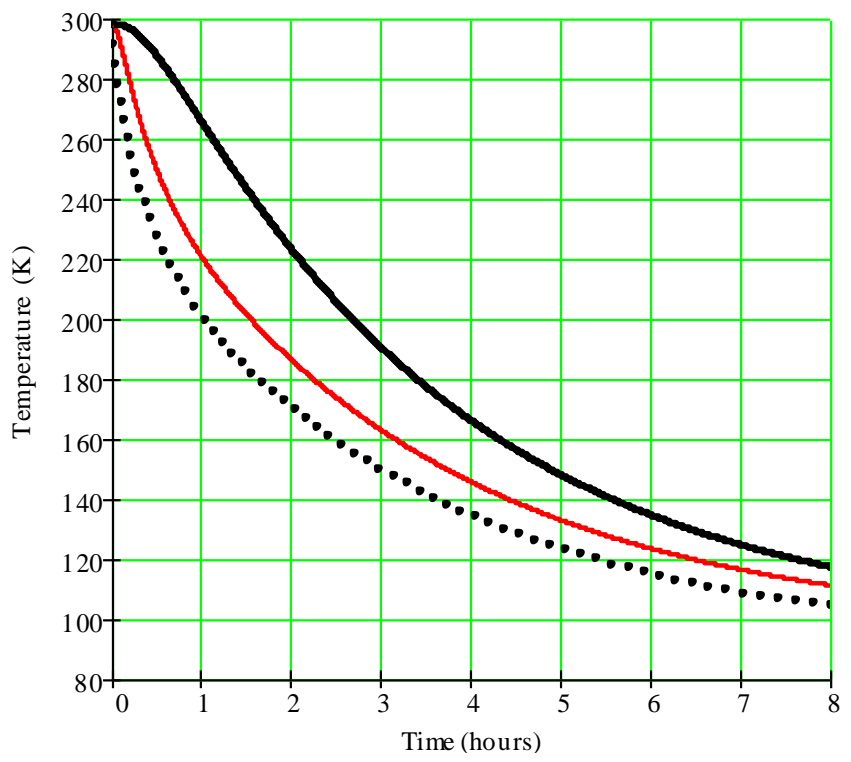

Fig.6. Calculated cooldown curves for HPGe detector (black curve), container for HPGe detector (red curve) and container for zeolite (dash curve).
TABLE IV: THE PARAMETERS FOR EQUIVALENT THERMAL MODEL

\begin{tabular}{|c|c|c|}
\hline Component & $\begin{array}{c}\text { Mass of } \\
\text { component }\end{array}$ & Equivalent parameters \\
\hline Cryocooler & - & $\mathrm{R} 0=33,3 \mathrm{~K} / \mathrm{W} ; \mathrm{C} 0=15 \mathrm{~J} / \mathrm{K}$ \\
\hline $\begin{array}{c}\text { Container for } \\
\text { zeolite }(\mathrm{Cu})\end{array}$ & $0,16 \mathrm{~kg}$ & $\mathrm{R} 1=950 \mathrm{~K} / \mathrm{W} ; \mathrm{C} 1=51,2 \mathrm{~J} / \mathrm{K}$ \\
\hline Thermal strap $(\mathrm{Cu})$ & $0,03 \mathrm{~kg}$ & $\mathrm{R} 2=6,2 \mathrm{~K} / \mathrm{W}$ \\
\hline $\begin{array}{c}\text { Container for } \mathrm{HPGe} \\
\text { detector }(\mathrm{Al})\end{array}$ & $0,12 \mathrm{~kg}$ & $\mathrm{R} 3=238 \mathrm{~K} / \mathrm{W} ; \mathrm{C} 2=84 \mathrm{~J} / \mathrm{K}$ \\
\hline HPGe detector & $0,53 \mathrm{~kg}$ & $\mathrm{R} 4=20 \mathrm{~K} / \mathrm{W} ; \mathrm{C} 3=167,4 \mathrm{~J} / \mathrm{K}$ \\
\hline
\end{tabular}

Note, the transient process of cooling is a sum of three components which are the exponential functions (8). The time constants of these components $\tau_{1} ; \tau_{2} ; \tau_{3}$ are determined by the roots of characteristic equation and allow to evaluate the duration and shape of cooldown curves (12).

$$
\begin{aligned}
& \tau_{1}=-1 / \lambda_{1}=3,4 \mathrm{~min} \\
& \tau_{2}=-1 / \lambda_{2}=21,8 \mathrm{~min} . \\
& \tau_{3}=-1 / \lambda_{3}=3,34 \text { hours }
\end{aligned}
$$

The cooldown curves for container with HPGe detector obtained sequentially after four warming out with an interval of 1 month are shown in Fig. 7. Clearly there is progressive degradation of the vacuum in the cryostat, which increases the duration of cooling. A comparison of calculated curve with obtained experimentally shows that the proposed model adequately indicates the real cooling process.

It should be noted that the growing divergence between calculated and experimental curves at temperatures below 140 $\mathrm{K}$ is related to the active adsorption of residual gases in the vacuum chamber by zeolite. This decreases the pressure in the chamber and heat losses. The cooling rate increases but final cooling temperature decreases at the same cooling power.

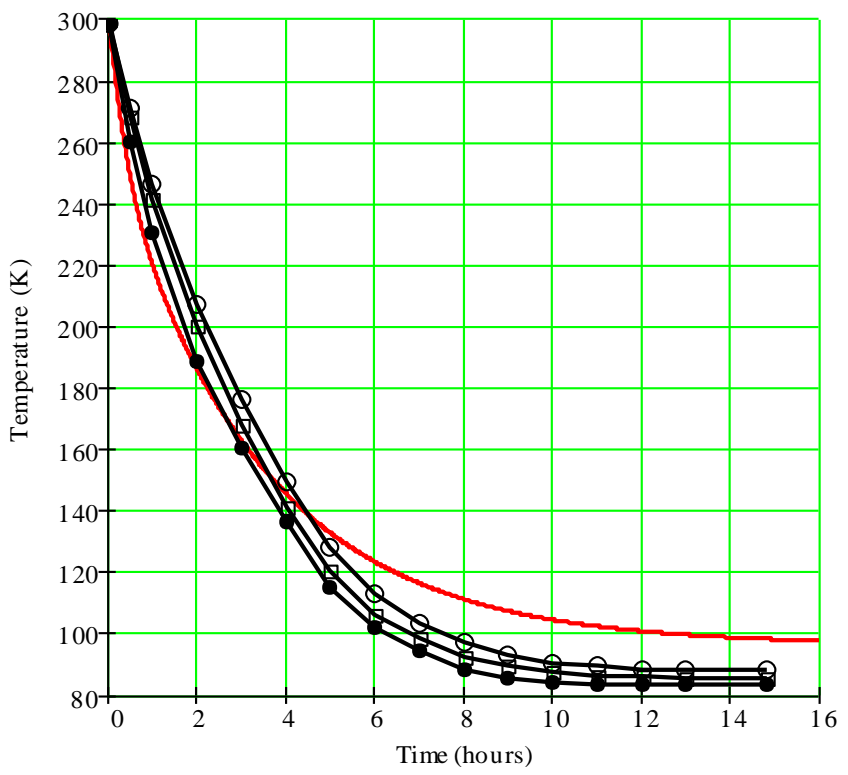

Fig.7. Cooldown curves for container with HPGe detector: experimental (black curves) and calculated (red curve). 


\section{THERMAL SIMULATION By SOLIDWORKS}

The equivalent model of cryostat proposed above is useful for the analysis of cooling as a dynamic process. However, it was assumed in this model that the temperatures at different points of the cryostat components, which are modeled as a capacitance as lumped element are the same. But the actual distribution of temperatures on surface of cryostat components has a significant influence to the analysis of heat losses. When parameters of equivalent circuit are determined the evaluation of thermal resistances of joints between elements in cryostat is particularly important [14]. The Solidworks Simulation was used for computer simulation of the temperature distribution in the joints between components of cryostat.

As example, the results of computer simulation of the temperature distribution at the junction between the cryocooler cold tip and tip of the flexible thermal strap are shown in Fig. 8 and Fig. 9. The diagrams for two forms of the tip: flat and cruciform are presented. In real construction, the flexible thermal strap provides the thermal contact between cryocoolers cold tip and detector container, Fig.4. For the simulation, the following parameters were used. The diameter of cryocooler cold tip is $14.2 \mathrm{~mm}$, the area of thermal contact equals 160 $\mathrm{mm}^{2}$. In numerical simulation, the heat load equals $2 \mathrm{~W}$ and was applied to the opposite face of the tip.

Note, that for good thermal contact is necessary to join clean surfaces with as high a pressure as possible. The most common material for thermal contact is copper, which forms an insulating oxide layer. This can be removed every time a joint is made by filing the surface. Pressure is applied by 6 screws for flat tip, Fig. 8 and 4 screws for cruciform tip, Fig.9. It is very useful to put a small amount of vacuum thermal grease between the contacting surfaces.

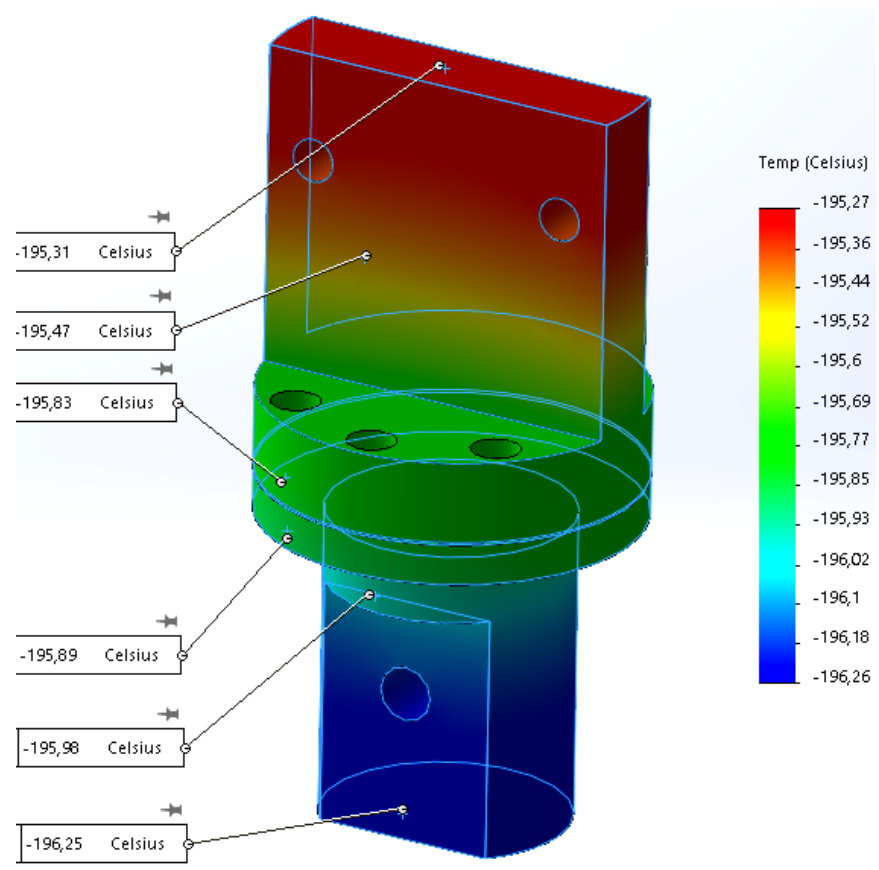

Fig.8. Temperatures distribution on joint with flat tip. Heat load equals $2 \mathrm{~W}$.

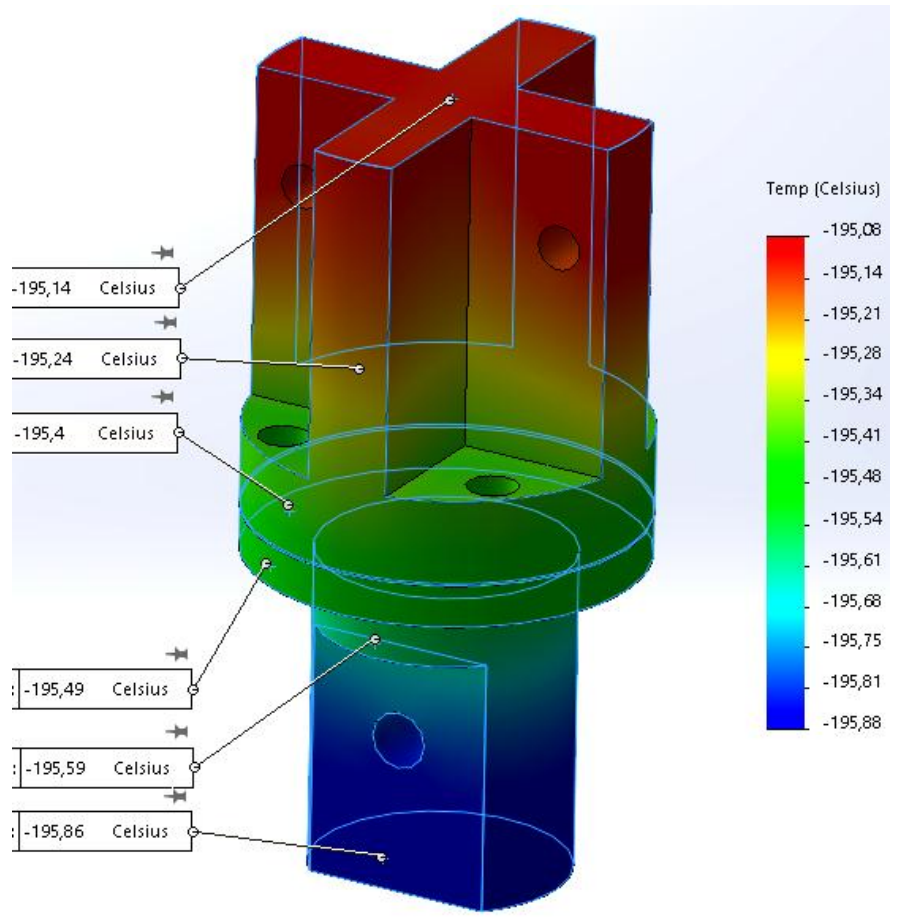

Fig.9. Temperatures distribution on joint with cross-shaped tip. Heat load equals $2 \mathrm{~W}$.

To reduce thermal resistance between contacting surfaces the thermal paste MUNG I (INTIVAC Corp.), consisting of silver powder blended into vacuum grease was applied. A thin layer of thermal paste provides a high coefficient of thermal conductivity equal to $18.2 \mathrm{Wm}^{-1} \mathrm{~K}^{-1}$. For calculations, the thickness of the thermal paste layer was chosen equal to 0.1 $\mathrm{mm}$. It is a real value, considering the cleanliness of interfaced surfaces and sufficient force of compression by mounting screws. As it is seen from the results of computer simulation, the total temperature drop on the joint is $1.0 \mathrm{~K}$ for the flat tip and $0.8 \mathrm{~K}$ for the cruciform tip (heat load equals $2 \mathrm{~W}$ ). Reducing the thermal resistance of the cruciform tip is due to increasing area of its cross section. Careful calculation and analysis of temperature drop on joints may indicate methods of reducing the heat losses reduce heat losses in the cryostat and reduce cryocooler power what is required for the HPGe detector cooling up to a predetermined temperature. Note that this problem is especially important for portable devices in connection with the small sizes of joints and thus the small contact areas between them.

\section{CONCLUSION}

The present article deals with a process of cryostat cooling in a portable spectrometer with a Stirling cryocooler. The simplified thermal model of cryostat as well as equivalent circuit for Stirling cryocooler based on the thermo-electrical analogies method are proposed. The parameters of equivalent circuit can be determined experimentally or calculated from data sheets. By comparing the load characteristics obtained experimentally for various cryocoolers and published in the open press with those calculated by means of the proposed 
model the sufficient accuracy of proposed thermal model for engineering calculations is shown.

In the framework of proposed model the process of cryostat components cooling as a transition process in equivalent electrical circuit is considered. This approach allows to use a well-known and standard methods from a theory of electrical circuits.

Undoubtedly the application of modern computer programs based on the Finite Element Method as well as network 2-D models with distributed parameters allows to calculate the process of cooling with more accuracy (as example [15], [16]). In this case, it becomes possible to take into account the nonlinear dependence of the specific heat capacity and thermal conductivity of materials from temperature, as well as nonlinear dependence of heat losses from the temperature. However, a simplified approach described in the article is useful for practical engineering applications to provide possibility of quick estimation the thermal performance of a cryostat.

\section{REFERENCES}

[1] M. Katagiri, A.Birumachi, K. Sakasai and K. Takahashi, "Portable Gamma-ray Monitor Composed of a Compact Electrically Cooled Ge Detector and a Mini-MCA system", IEEE Transactions on Nuclear Science Symposium, vol.50, Iss.4, pp.1043-1047, 2003

[2] K. M. Yocum, G. N. Martin, J. F. Colaresi, and H. S. Miley. "Improvement in Ge Detector Cooling". Proceedings of the 30th Monitoring Research Review: Ground-Based Nuclear Explosion Monitoring Technologies, Portsmouth, pp. 824-833, 2008.

[3] E. L. Hull, R. H. Pehl, J. R. Lathrop, G. N. Martin, R. B. Mashburn, H. S. Miley, C. E. Aalseth, T. W. Hossbach, and T. W. Bower. "Mechanically Cooled Large-volume Germanium Detector Systems for Nuclear Explosion Monitoring". Proceedings of the 28th Seismic Research Review: Ground Based Nuclear Explosion Monitoring Technologies, LA-UR-06-5471, Vol. 2, pp. 822-831, 2006.

[4] R. M. Keyser and R. C. Hagenauer." Performance of a Portable, Electromechanically-cooled HPGe Detector for Site Characterization". Journal of Radioanalytical and Nuclear Chemistry, vol. 277, Iss. 1, pp 149-153, July 2008.

[5] Randall F. Barron. Cryogenic Heat Transfer. CRC Press, 1999.-392 pages

[6] Yeon Suk Choi, Dong Lak Kim and Dong Won Shin." Estimation of Cooldown Time in Cryocooled Superconducting Magnet System". Progress in Superconductivity and Cryogenics, vol.12, No.2, pp.25-28, 2010

[7] Seong-Je Park, Yong-Ju Hong, Hyo-Bong Kim, Deuk-Yong Koh and Yang-Hoon Kim. "Performance and Reliability Characteristics of the Free Piston Free Displacer Stirling Cryocooler". Journal of the Korea Institute of Applied Superconductivity and Cryogenics, vol.6, No.2, pp.46-51, 2004.

[8] D.L. Johnson, I.M. McKinley, J.I. Rodriguez, H. Tseng and B.A. Carroll." Characterization Testing of the Thales LPT9310 Pulse Tube Cooler". Cryocoolers 18, edited by S.D. Miller and R.G. Ross, Jr. International Cryocooler Conference, Inc., Boulder, CO, pp 125-133, 2014.
[9] S. Kim, E. Holliday, R. Unger and R. Wiseman.” Development of CryoTel DT Cryocooler". Cryocoolers 17, edited by S.D. Miller and R.G. Ross, Jr. International Cryocooler Conference, Inc., Boulder, CO, pp. 101-105, 2012.

[10] M. Mai, I. Rühlich, A. Schreiter and S. Zehner. "AIM-Space Cryocooler Programs". Cryocoolers 16, edited by S.D. Miller and R.G. Ross, Jr. International Cryocooler Conference, Inc., Boulder, CO, pp 133-141, 2011.

[11] Allan R Hambley. Electrical Engineering: Principals and Applications. 5 th Edition, Prentice Hall, 2011, 893 p.

[12] HandSPEC Hand Held HPGe Spectrometer. Baltic Scientific Instruments [Online] Available:

http://bsi.lv/en/products/hpge-detectors-spectrometers/handspec-hand-held-hp ge-spectrometer/

[13] R. Smith. “ Camera Thermal Design”. [Online] Available: http://www.oir.caltech.edu/twiki_oir/pub/Keck/NGAO/DetailedDesignR eviewNIRTTS/KAON902_TRICK_Thermal_Design.pdf

[14] D.L. Johnson, and R.G. Ross, Jr. " Cryocooler Coldfinger Heat Intercepter". Cryocoolers 8, edited by R.G. Ross, Jr. 8-th International Cryocooler Conference, Colorado, pp 709-718, 1994.

[15] J.Z. Jordan. "Network simulation method applied to radiation and viscous dissipation effects on MHD unsteady free convection over vertical porous plate". Applied Mathematical Modelling, vol.31, 2019-2033, 2007.

[16] F.DEL Cerro, A. Campo and F. Alhama. "The Teaching of Unsteady Heat Conduction Using the Thermo-Electric Analogy and the Code pspice. Nonlineal Models". International Conference on Engineering Education and Research "Progress Through Partnership". VSB-TUO, Ostrava, ISSN 1562-3580, 79-86, 2004.

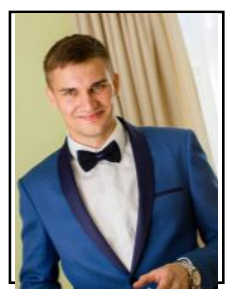

O.Yakovlev was born in Latvia, Balvi on February 8 , 1992. Master of science in Engineering Technology, Mechanics and Mechanical Engineering. Scientific directions: engineering mechanics from 27.06.2015 given by RTU Faculty of Mechanical Engineering, Transport and Aeronautics. RTU PhD student in Engineering Technology, Mechanics and Mechanical Engineering. Scientific directions: engineering mechanics from 1.09.2015.

Baltic Scientific Instruments company Mechanical Design Engineer from 13.01.2014. RTU Faculty of Mechanical Engineering, Transport and Aeronautics researcher from 12.05.2016. RTU Faculty of Mechanical Engineering, Transport and Aeronautics lecturer from 1.09.2016. Researcher in the area of nuclear physical equipment problems. Mechanical design engineer of numerous physical equipment projects for countries with nuclear power and project for space applications.

Kondratjev, V., Gostilo, V., Owens, A., Yakovlev, O., Vība, J. Vibration Characteristics of Miniature Stirling Electric Coolers. Vibroengineering Procedia, 2016, Vol.8, pp.409-413. ISSN 2345-0533.

Yakovlev, O., Vība, J., Gostilo, V., Efremova, N. Simulation and Design of Radiation Shielding and Collimation Systems for the Precise Gamma-Spectrometric Equipment. In: Proceedings of International Conference on Innovative Technologies, Czech Republic, Prague, 6-8 September, 2016. Rijeka: Faculty of Engineering, University of Rijeka, 2016, pp.23-26. ISSN 1849-0662. 